\begin{abstract}
II.
Fiinfte Fortelzung

des Verzeichna/Jes der vom Himmel gefallenen Mafen;
\end{abstract}

nelfil weitern Nachrichten von einigen fchon bukannten und yon neuern Feuermeteoren,

E. F. F. ChLAdust.

(Mil Zuralzea roa Gilbert) ')

1. Nachrichten von Meteorfiein - Fällen.

1421 fiel auf der Inlel Java ein Meteorftein her$a b$, von der eine dortige Chronik folgendes meldet: „Bei Gelegenheit einer Verfummlung der Oberhäup-

-) Dafs wir Hotruup baben, das ausfibrliclie, von Herrn Chladui auf leiner letzten Reile angnearbeitete Werk über Feucrmeleore und die mil drafelben hesabgefalleaca MaITeu im Verlage einer Wicner Buchluanillung im Drurk zu erbalten, wird diefen Nachiragen, welche leide 5 fruhern in den Annalcu belianut geniachien Aufficze zu ergänzen heliimme find, nichs an Interefte fir meine Lefar, wie ich glaube, brochmeu. Auch habe ide te mit einigen nicht univichtigen Zufatzen veruehre. Gill.

Ainul. d. Pligfik. B.63, Sı, 1. J.181g St.g. 


\section{$\left[\begin{array}{ll}28 & \text { [ }\end{array}\right]$}

ter zum Leichenbegingnifs des vorftorbenen Stulians, und zum Regieruugsantritt des Pangeran Tranggana (1421) erhob fich ein lelirecklicher Sturm mit Dunzner und Blitz (in der Sprache alterer Chronikenfelıreiber ein Fenerneteor mit Getöfe). Ein junger Menfch, Namena Jaka Sifóla, ging ans der Mofcher, um nach dem Wetter zu fuhen, da fah er einen metcorifchen Stein neben fich zu Boden fallen, der ilnn aber nicht belelıudigte. Der Stein ward dem Sunna Kali Jaga gebracht. Man dankle dem Allmachtigen für den von der Mololinee abgewendeten Schaden, unaclite cine Ablildung vou dem Sleine und ftellı: fie ann Thore auf der Nordleite aus ${ }^{\bullet}$ ).

( 1552 den 19. Mai. Dic von dem grofsen MLetcorftcin-Falle bei Schleufingen in T/utringen von Spaugenberg in lieiner Mangfeldifchen Chronit als Augenzengen gegeloene Nachricht, ift von Herrn Marcel de Sones, der überhanpt viel Unrichtiges iiber Meteorficinfalle gefagt hat, felis verunftaltet worden, indem er Schleufingen, wclclıcs or nicht kinnte, nit Schleishein, bei Mïn-

7) Sir Thomat Stamford R a fli en Ififery of Java. Londoll 1917 8. Vol. 2 P. 237. Flr. Gelt. Reth von Simemerring iu arünchen hat inje ho nuf diefe Stelle aufinerkfaw gemachs. Der Yerfates war wilhreud des Kriege bristifecher Stutuhalier ron Jasa, und if es jetzt ron Fart Marlborough auf Sumatra. Befonders merkwrirdig laud die vielen rou ihm befchriebenen und obgebildeten dortigen t'eberrefie alter

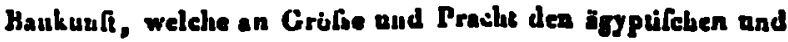
indilchen nichs anchlieben. Chl. 


\section{l 19 J}

cluen, wo er die bekannte Bildergallerie gefehen, verwechfelt, und das Lireignil's nach Baiern verfetzt hat ").

(?) 1572 den 9. Januar ein Steinregen bei Thorn *), mud 1740 oder 41 ein Steinfall in Grön-

-) Diefer Itrthum in den Ann. de chimie t. 85 p. 278 if auch in Tilloch's philor, magaz. Sept. 2814 wiederholt worden. Aehnliche Verirrungea fand ich in einem der vorzüglichera englifchen Joura ale, wo bei Erwähunug der kleinea Landkarten in des Hrn, von $Z$ a ch monatliclier Korsefpondens, unter welcher: Seeberg bei Gothe, lieht, bemerkt wird, diefer Mir. Seeberg mülte a mofl excullent gє0Erapher feyn, weil er to gute Kartes luefere, ingleichen in der Gasefte de France 180y, wo ron einem auf der Olifee durch Sturn hefchidigten Schitte die Rede war, welches am Schlepptau gefülurt werden mufte, und aufiatl : on $l_{4}$ conduit \& la remorgue, gefagt wird: On l'a conduit asehbepe tau. (l) Chl.

-) In I. H. Zernecke Thornifchen Chronika (ate AuA. Berlin 1787) heilse es S. 157: "Aneo 1572 den 9. Januar, als die Weichfel 3 Tage blutfarb gewefen, nad wiederumb ihre recbic Earbe bekommen, ift su Thore in Prenfsen uin 9 Uhr is des Nacht ein sreuliclier Wolkenbruch eatlinoden, dafs durch deafelben Wattergafo ein grofo Theil der Stadtmauer hernieder gefallen, 19 Joch an der Brneken hisweggefuhrt worden, und bei 300 Meolchen ertrunken find; mit hiazu hat es topfindig: Steine gehagelt, die viele Luute za Tode gelchlageo und cin Fouesfirahl roos aimmel hat der Stadt Kornhaus verbrennt. Aus Sebaft. Munfleri Cosmographia lib. V. p. 12go." Er fetzt hinzu: "In den MStis Thornienfibus finde davon gar keine Notasn, halle alfo da-

B. 


$$
\text { [ } \cong 0 \text { ] }
$$

land $*$, berulien beide auf apocryphen Naclirichten.

Von den folgenden beiden bisher von nir nur ganz kurz erwilnnten Steinfüllen in Italien giebt Soldani in den Memorie dell' Accademia di Sie. nn tomo IX p. 8 und p. 219 unftindliciste Nachricliten:

1697 den 25. Jan. in der 23 ften Stunde, (alfo et-

für, dafs diefes aus dem grofsen Buch der kleinen Wahrlucis mufs genominen feyn." In clem Falle, dafs an diefrr Nachiricht, dic icb Itrn. Gch. Rath von Sümmeriog verdanke, Joch folltr etway Wabres leyn, fo künule es viclleiclat ein grofses Fcucrmeteor mit eiuem Steinfalle und mit ciucm Niederiall ron vielem rollen Siaube in den ohetn Wcichrelgegendia gewrfen fegn, bei dena fich zuglcich auch ejue grofse Mluge Walfer niedergelchlageo hälte, etwa wie 17 t.6 llen 2. Jun. bei Tuam in Irlend.

Chl,

-) Der ungeheure Sicin, welcher nach Egeile in Grünland in einer Wintaruachl 2 wifchen 27 f́l uad $274 \mathrm{t}$ foll herabjefollen feju, (Annal. B. b3 S. $37^{8}$ ), war, kic Hr. Berörath Gielecke mir lagt, kein Meteorftein, fuudern cin Felfenftuck, das lich ablofie, uod von einem hohen Berge weit in das Thal hernbrollec, 20 Jacobshasn nnter $69^{\circ} 46$ Breite. Das Stuckeheu, welchry er mir ron diefern Stcine gegeben hal, if aichis auciern, ols ein weibslich grauor Grünfiein. Er verfiehert, dafi mehrere Mal die Crün]änder von Solchen Lerabjerollten Steinfu gefazt habed, fie wärea rom Himmel gefallen, und hat mir anch eiu siück vop einesn glimmerartigen Steine gegelien, welcher unch den Auslagen der Gı̈̈nlauder uater $\left(15^{\circ}\right.$ \&' Brcice lierabgefallen feya full, oder vielrachr ron eioem Berge herabgerollt ifr.

Chl. 


\section{$\left[\begin{array}{ll}21 & 1\end{array}\right]$}

wa zwilchen 4 und 5 Uhr Nachmittaga) hörte man bei Siena viel Gerüuch wie 3 Kanonenlchüfle, und noch anderes Zifchen und Platzen, wie viele Racketen, und ein Saufen, wie von einem ftark brennenden Kamin, faft $\frac{x}{b}$ einer Stunde. Es entfrand eine grofse Finfternifs, und ein Rauch, der nach Schwefel rocll. Es fielen Steine nieder; ciner 13 Unzen fchwer, nicht weit von dem alten Galthofe bei Pentolina, machte cine Vertiefung in die Erde cine Palme tief. Er war wïhrend des Falls mit vielenı Rauch umgeben. Man fand iln lieils und nach Schwefel riechend. Er war wie ein Eifenerz und answendig fchwärzlich. Anch noch andere Steine ficlen bei Menzano, Cipraja, al Padule. - Dicfes ift aus einem Auffatze von Pirro Gabrieli, Prof. flor in Siena, in den Memorie dell Accademia de' Fifiocritici No. 18. Hierauf folyen S.9 noch alldere damit übereinfimmende Naclirichten aus dem Briefe eines Ungrnannten an Pirro Gabrieli.

$179^{2}$ den 17. Mai liörte man bei Caftel-Berardenga in Toskana deg Morgens um 8 Ulir (mach ital. Zeitreclumung, alfo nach der unfrigen etwa zwilchen 5 und $;$ Uhr) in dem ganzen fidweftlichen Theile von Toskana, melir als 100 ital. Meilen weit, Getöfe fturker als eine Kanonade, und hernach einige Minuten lang Getöle von anderer Art. Einige filien cine grofse dichto und feurige Kugel, die nach ihrer Explofion vielen Rauch und einen Streifen znrückliefs. Der Himmel war heiter und blicb es, das Licht der Sonne war aber matter, wegen einer Art 


\section{[ $: !]$}

von Nebel, dor fich hermach zum Theil verzog, aber einige Tage lang noch etwas bennerkbar war. Es fielen Steine zur Erde, von denen dor Patrizier Gal. gano Saracini Lucherini einen bel'afs.

1810 im Augult (dér Tag ift niclit angegeben), fiel um die Mittagszeit in der Graflchaft Tipperary in Irland oin kleiner, nuch in dielen Annal. (B. 6o 8, 256) erwähnter Meteorftein herab. Gename Nach. richton davon giebt Analyfis of tho meteoric fone, which fell in the County of Tipperary, by Milliam Higgins, Esq. (Dublin 2811, 8.) ein Scluriftchen, welchen nebft einem Stüoke des Stcins, (fo wie von dem 1813 den, 20. Sept. bey Limerick gefallenen) ich der Gefälligkeit dea HIn. Prot. Giel'ccka verdanke. Nach dem Bericht von Maurico Crosbie Moore, Esq., auf defen Befitzung der Stein herab fiel, hörte man ein donmeraln liches Getölo und ein Zifchen in dor Luft. Ein Arbeiter, nalse an Wolınhaule lah ein kleines Wölkchen (aus dem das Meteor umgebenden Rauch und Dampf beftehond) welches fioh ander's bowegto, als die anderu (eigentlichen) Wolken, und woraus ein Stein lchnell über dio Köpfe der Zulichaner gehond auf oin Feld, etwa 300 Ellen rom Haulie, niedorliel, und einen Fufs tief in dio Erdo einfichlug. Er war fo heils, dafs er erft a Stuudon nachhor mit den Hinden berilirt weiden kounte. Er wog 73 Pfind. Die Geftalt war falt kubifclı; an 2 Soiten find dio Ecken und Kanten abgorundot; an 2 andorn find Einbiobungen und Ausbiogungen. Higgins fand bej oi- 


\section{$\left[\begin{array}{lll} & 25 & 3\end{array}\right]$}

ucr Analyfe darin folgende Beliandtheile: Kiefelerde ;8,25; Eilén 39; Magnufra 9; Scliwefel 4; Nickel 1,75 $=102$. Bei der Analyle eines andern Stücks fand er Kiefelerde 46; Eifen 42; Magnefia 12,25; Scliwefel 4; Nickel 1,50 $=105,75$, wo der Ueberfchuls von angehängtem Sauerftoff herrührt. Das Eifen enthicit keinen Kolnlenftoff. Der Stein (oder nach Ablchllagen einiger Stücke, ungefähr die Hälfte) befindet fich im Irilchen Muleun zu Dublin. Hr. Profeflor Gielecke, der mir dicles lagte, zeigte mir cine Abformung des Steins in Gyps, und Hr. Direktor von Sclireibers eine Kupfertafel, die Sowerby in London liat ftechen lafen, worauf diefer Stein nebft den von Yorkfhire (1795) und den von High - Poffil (1804) in natülicher Grölse dargeftellt find, In den von mir gelelienen Stïcken ift das Inmcre etwas gleichförmiger dunkelafclıgrau, als bei den meiften Meteorfteinen, nit inliegenden Theilchen von Gediegen-Eilien und von Schwefel-Eileun, und wenigen kleinen braunlich granen Körnern. Die Rinde ift Icliwirzlich, oluse Glanz und etwas rauh. Ich finde die meifte Aelunlichkeit mit den Steinen von $\mathrm{Li}$ merick (1813). In beiden Stcinarten zeigt fich anch im Bruch das Eifen an manchen Stellen als ein metallifch glinzender Anflug, fo wie anch an manchen Steinen von Laigle $(180 j)$.

? $181 \tilde{j}$ in Somner, (olnne Angabe des Tags), follen um 1 Uhr Nachnittags, bei Mralpas, 15 engliiche Mrilen von Chefier, viele Stcine, mit Gewitter (Feucrerlcheinung und Getöle) aus einer lich- 


\section{[ بf ]}

ten Volke gafallen feyn, dio anfangs weich und fehr heifs gewefen, aber hernach hurter geworden find, nach einem anonymen Bericht, aus einem Provinzialblatt, in 'Thonfons Annals of Philofophy, Nov. 1813 p. 396 . Hr. Direktor von Soliroibers, der mir diele Notiz mitgetheilt hat, lagt mir, dals den Englifohen Phyfikern nichts weiter davon bekannt geworden foy, und man die Richtigkeit der Sacho bezweifele ").

Der Fall eines in Annal. B. bo S. 254 crwihnten Steins im Dorfe Slobadka in Gouvernement Smolensb hat fich nicht am 11. Juli, fondern am 11. Augalt 1818 (odor don rg. Juli a. St.) ereignot, nach den Nadhrichten, dio ich im Hamburger Correfpondenten No. 158, und in Schwäb. Mlerkur No. 243 fand.

[1819 den 13. Juni find zu Barbezioux, im Dopartejneut der untern Cliarento, Meteorfteino unter den gewöhnlichen Erl'cheinungen herabgefallen, laut eines Schreibens eines Hrn. Andrieux, welohes $\mathrm{Hr}$. Biat wahrond moiner Anwefonhoit in Paria, in des Akarl, dor Willenfehaften vorlas. Man erwartete Praben derfolben derfolban aus chemil'chen Prüfung.

Gillert.]

Dor von mir in Annal. B. 54 S. 334 erwihnte Stein in dom Krönungsftutle dor Konigo von Eng* laud ift, wio man mir fagt, kein Motoorftein. Da.

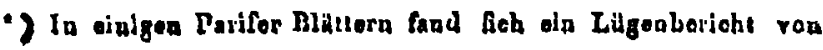
nlaem Stoinfalle la Juilly (Dep. do Scino et Marne) am iaten Juli iths, wolchor aber bald nacherex widercufen warlu.

CN. 


\section{$\left[\begin{array}{lll}2 & \mathbf{5}\end{array}\right]$}

gegen reden aber einige Blitter von einem nerkwïrliggen Steine, der in einem Gewölbe zu Dunfinan in den Ruinen von Macbetlis Sclilofie foll fi:yn gtfunden worden, (?) und von welchem dio Vermuthung gexulgort wird, dals er möge ein Mcteorftein gewelion leyn.

Bei dem 1815 den 3. Oktober bey C/anfigny, nicht weit von Langres gefallencn Steine, der keiuen Nickel enthült, und im Anfelien fich fohr vou andern unterfelicidet, kommt mir einiges lehr problematifch vor. Ich l'elıo darin mit blofsen Angen und noch moln mit der Loupo folur klcine metallileclı Theile, cben fowohl wic in anderu Metcorfteisen, wiewoln in fehr geringer Mengo. VFenn dicfie Gediegeneifen find, l'o widerl'priclıt diefos der gewöhnlichen Belıuptung, dal's Niclicl immer mit dem meteoril'chen Gediegen-Eifon verbundon ley. Siıd lic aber Scliwufol-Eilen, fo widerlporiclıt dieles der be:lunptung von Vauquelin, dal's diefo Stcine keinen Scluwefol eullalien, lie verdienten allo wahl noch cin Mal genan in dicler Hinficht chonilicle unteslinglit zu werden").

7. Beitrige aur Kenalnifs mancher Gediegen-Eifea-M.LI:a, An oinem Stïcke Gedi.ygen-Eifen van Tuluca in Mexilo (Annnl. B. 56 S. $58 i$ ) in der Kailorl. Natu-

-) Diefen Ergïnzungen fïge ich hier qoch zwei ,bei, nus eiacra Briefo eines Itr. I'a uli in Bruguatelli'a phyith, chemi- 


\section{$\left[\begin{array}{lll} & 2 & \end{array}\right]$}

ralicufannulung zu Wien, fand ich an eincr polirten und geitzteu Fliche von ungefuhr \& Quadratzoll Grölse, auch die Widmanftadtil'clien Figuren; es ift aber auffallend, dals die Streifen niclit, wie bei andcrm Meteoreilen, in 5 Riclutungen, londern nur in

Scher Zeitelurift. Wahreud Dr. Bulfi heilst es in dielem Bricfe, Joh. A adreas von Pral o' Chronik durchlief, ura eine Erganzung zu Cluladui'v Verzeichnifs von Meteorolitheu (ill Th. i S.515 jeuca Journals) fortzufotxun, bing ich in derlelben Abficha die IVerke fu uiluerer berülumer SelurififielIer Inaliens durch, und ueiue Nachfuchungen find nirhe fruchilar geblieber. Camillo Lconlas rili iu feinem Speculum lapirum lib. I cap. 5 lige, nachdenn er von der Entliehung ler Steiue in ler Erde, und in Thieren elc, gefprochen liat El non Jalum in his luais lupieles ganerantur, yarum etiam ot in ä̈re, ficut habetur a philnfophis, et maxime ab illo fummo philofopho ac nofaris temporihus monarra, praereptore meo, Domino Gaetuno do Fienis in Commento metaurorum, in fine fecunti trautatus tertu libri, qui dicit: lapilles ganerari polfunt in aere, cum exhalativ habet partes groffas terreas admistas cum humiditate grofja viscofa. Et rejolutis partibus magis jubtilibus, et terr-fribus cundenfut is a calidu, fit lapis, qui ratione fuat gravitatis ad terram defiendit. No/iris temporibus in partibus $L$ ombardiae lapis magnat quuntitatis ex nubibus ceridit. Genanates Werk if von abry, aus welcher Zeit kein bedeutender in der Lombardei herabuefalle-ner Aerolitb bui audern rortiöm rnt. Die Aerolithen von 1458, $1492,1+132$ uad $1+96$, in Chlaclai's Verzeiclatifs liul von ilın rerichiedell, Deya Lcouhardi histe von dir feıa wohl nicht ligen kïnaed: nofiris tempuribus, und theils fielen tie au auderu Urien herab, lheils warea en mehrete, judels Leuuhardi aur von ciuew ciuzigen, lehr grolsen Ster- 


\section{[ 27$]$}

zwei Richtungen oinandor ziennlich rochtwinklig durchkrenzen.

Yon der in Ungaru im Sarofeher Comilat bei Lenarto gefundenon Eifinmalle (Annal. B.49 S. 181) befindet fich das molirero Pfund Iclivere Stïck, wel-

a Priche. Nach Prof, Arignole iff Lnonhardi's Werk alter als das von Georg Agrieula, und tie Eilure zuerff ejuse Mineralogie geliefert zu haben, gehörtc folglich mainem $V_{a-}$ corlanile. - Finen aodern noch in keinern Verzeichniltu sufgefulurten Metenroliphen Inde ich iu dea Actis Eruditorem b. 7 Suppl. p. 235 belahrieben, wie colgt: Deforptiv mefeori ignili ah Flenrico Burham in Jamaica, anno ;-ou ohjervari, (ex Trans. angl. a 1718 v. 357 p. K37 et 838 excorpl. et in campend. redact.) Fillit objiervator globuen isneum mole aequalem globis ferreis pulpert nitrato ripletis, quos bombas vocare folont, mole admodum celeri per aevetu ducielentem, qui fulzure prorfius infogui ennieabat. Cumpres ad locum accedoret, ubi terram attixertat, varios ihidem ub-

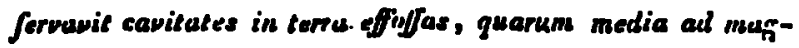
nitudinem cranis hunani, quingue vero aut fes minores circumcirce ad pugui majnitudinem accerlebant. Tanta wat profundifar, ut baculis, qui fpectantibus ad monus ersint, explorari non polfet. Gramina eirce cavitates affo/fus cremata confpicivbantur, of odor julpheris percipiebatur per aliquot tomporis intervallum. STocse prascedente inbres cumitata fuerant fulgura crebriopa eum conitra vehementiort. Ohno Zweirel hälle man beim Nachgraben Meteorolitbren gefunden. - Noch erwähne jeh hier cines fonderbaren Regens einer Erde, die gave rom Magnes angezogen wurde, welcher am 21. Mai 17.57 auf dem Adriatifehen Mecrelawilehen Monopoli und Lijfs herab firl, and ron Joh. J acob Zaa iebelli beichriehen worden if, So woil Er. Dr. P^oll. 


$$
\text { [ } 28 \text { ] }
$$

ches der l'rofefor Sennowitz'in Eperies befufs, jelet in den K. K. Naturalienlibinet zu Wien, wo ich es gelelun lıabe. Die Widnunfiadtifclien Fignren zcigen lich darauf lohr l'chün auf geitzten Flyclicn, auch ift das octinedrifcli-kryfiallinifche Gefïge in Bruclı und auch an dor hier und da geftrickten Oberflüclie deutlich zu lélien. Dafo dicles Eifen Nickel enthuilt, erluellt ganz beflinunt aus einigen vou Hrn. Basou von J a cq ui $u$ angeftellten Luterfuchungen. Merkwärdig ift, dal's fich klcine Theilrlucu, and belonders, ein inliegendes elliptiliclies Siück einige Linien im Durchneffer von anderer Farbe und Glanz auf einer abgelüg!en Fluche beinerken lafen. Ob diel'es Nickel oder Scluwefol-Eilin, oder was es fonfl fey, wird fich bei weilerer Unterlinchung wohl ergebur.

An diefen Eilen l'owohl, wio an den Mafen von Agram und von Elnbogen, zeigt fich auclı Schwefel-Kifen in cinigen Klïften und Spalten, welcho zu Theil damit ausgefillt find, nud wodurch das Schweil'sen folchen Eilens an dergleichen Stellen bi:hr erfclivert oder verlindert wird. Auch fah ich bei Hrn. von Sclureibers 2 längliche abgerumete Siticlie von Schrvefel-Eilien von einigen Linien Durclınefler, diefich in Pallas il'chem Godiegen-Eil'en aus Sibirien befunden latten ").

-) Dafs Hr, Laugier bei Seinor Analyfe diefes Eifeas darin 5 I'raceat Schwefel fuad, welche allen, dis: ror ihm Pillasiliches bilen zerlibt hatlen, entgangon warea, (Anial. istS 


$$
\left[\begin{array}{lll}
-9 & -9
\end{array}\right]
$$

Aus den Eifen der Elnbogener Male, (dem verwünfcliten Burggrafen) befitze ich jelzt durch die Güte des Hrn. von Widnanfiudten einc Federmeferklinge, welche durch blaues Anlanfen mit den von ilım cntdeckten Figuren l'cliön damal'cirt ift.

In Thom l'on's Ann. Sept. 1818 p. 2 in wird bei Gelegenheit des gefundenen grolsen Stücks liatin, 1 Pind g Unzen l'chwer, auch ein Stück Gcdiegen-Eilen erwähnt, welches He aland in London befizt, und das an der Küfte von Umoa, in der Provinz Honduras, 10 cmglil'clie Meilen vom Meare, ift gefunden worden, wo mchr dergleichen $\mathrm{Ei}$ lin leyn loll.

Von dell an der nürdlichften K:j/ie der BaffinsBay, nach den Reife-Nachrichten der Expedition unter Kapitain liol's frcle findenden 2 Klumpen von Cediegen - Eiten, ans dem dic Einwolner eine fehr unvollkommene $\Lambda$ rt von Mefiern machen, werden wir loolfentlich bald genauere Niachuichten erlialten ").

In Steiermark ift auf eineun Berge bei Cilly cine

St. 2 S. 182), verliert durch diefe Bennerkung alles Aoffallente. Fis liat, habe ich alun reche vorfiualca, das Eifen von del olivinartigen frciuigen Eintuengung Jurch Eehandtiln der Malle mit Salpeterfäre völlig zu trennen gefucht, und die Aualyle drei Mal angefiellt. Cill.

-) Nach Hru. Brazde's Aunlyre enchäle das Eifen diefer Celir unvollkommenen MeIter Nickel, Mebr davon künftiog. cilbert. 


\section{[ [ ju: ]}

Eifenumafte gefiunden worden, die Itr. von GadolIa (jetzt Deputister von Cilly) befitzt, und welche nach Alffägen einiger Stücke, von denen ich auch cines durch die Gefilligkeit neines Freundes, des Ylerm P'a lu l'artl'ch erlalten habe, noch.ungefilln 16 l'fund wicyen kann. Dicles liilen zeichnet fich dudurch aus, dals damafcirte Figuren, den Vidnunftidtifclsen alnnlich, mur etwas feiner, fich itberall fechon ohne Actzung zeigen *). Ohngeachtet dieles fo ansgezeichuct kryftallinilchen Gofiiges, nud obgleich es fich kanm begreifon lifist, wie cs nuders als durch Hernbfallen auf einen Berg, wo keine menfchlichen Wohnungen und keine Eilenhüttein find, l:önne lingekommen feyn, hult Hr. von Widmanfizadten es doch'nicht für metrorifch. Und iclı frimne ilın darin lsei, ans folgenden Gründon: 1) Weil es keinen Nickel enthält, nach den Unterfuchnngen, die Hr. Prof. Scliolz in polytechnilchen Inftilut in meiner Gegenwart angeftellt laat, und zwar nacle Wollafion's Methode, mit dem wirkfamften Reagens, deun blanlauren Eilenkali, (oder Blullauge); welches bei Cegenverfichicn durch Hinzufigung einer faft unbeftimnbar kleinen Quan-

-) Zwar gielut fich noch bei; manchem noilern Eifrn ader Stshl das kryflathinifche Gefuge bei deun Aetzen, nach ding bekanntep Verfuchen ron D.unirll, durch ha:llere und dunklers Sircifcn zu erkeanea, aber wullt bei keillem in deu, Grade, wie bei dem hier ernäbuten felion oitne Aetzugg.

Chladni. 


\section{[ $\left.\begin{array}{ll}51 & 1\end{array}\right]$}

titüt von Nickcl-Aun̈̈ung zu folur vieler EifenAuföfung fich (E)hr bewihn't zeigte. 2) Weil dieles Eilen fpröder ift, alo gewölnulich das metcorilchlı Eifen. 3) Weil dic Geftalt der Mante, welche ich bei Hrn. von Gadolla lah, nicht lo befchaffen ift, wie fie bei einer Mcteomnale l'eyn nuilfste, l'ondern fo parallelepipedilich, mit faft geraden, ziemlich einen rechten Winkel mit einander machenden Fliiclucu, dafs fie in einer Forn gegoffen zn feyn fcheint, ungefilir lo: wie die angcblichen Stücke des Grol's - Cansdorfer Eilens in Dresden und in Freilserg, welche indefen liein lolches kryftalliniliches Gefïge zeigen. Der Kolulenltoffgehalt, welchen Hr. Protelfor Scholz darin gefunden hat, lpricht auclı nicht für einen neteorifchen Url'prung, Von falılackiger Subfianz ift nichts daran zu benicrken ').

-) Ebeofills ein Product irdifcher Schunelzarrg, und keineswege ineteorifeh, if las durelı feiu Vorkoumen elwas prublemalufche Eifen, wovon ficle im Bulletin de la Soc. philomal. ${ }^{517}$ P. ${ }_{17}$ Wachriche aus einem in der Aladersio der Wiffenfehafien 20 Paris gelefenen Aufraze roo Heaty, (riirecteur des ponts et chauffeses,) findet. Wiefe Malle ron Eifenoxyd, geinengt tnit Portionen vou Gediegen-Eiled, lag bei Florac, (Departenuent de la Loxere) in dein Betie eiues Gebirgswalleru, war 5 Decimcler lang, 5 breir and $y^{\frac{1}{z}}$ bis 2 dick, wing uagefahr 150 Kilograminen (joo Pfund) war cifïrnig, an der Oherbinche rauh und angultaltet, felien rom WnITur gerollı zn feyn, und zeigle aaswendig Einchücke ion Nufelucl-Verfeincrungen. Da Indere war blafig, an mau. 


\section{$[5 \div]$}

[Folgenden Zufulz verdanke ich Hrn. Brongniart in Paris. Der Profetror der Mineralogie $N_{0-}$ rodecki zu Wilua, luatte ilum im J. 1818 ein kleines Stück einer Eilenmafle überfchickt, welche man in dem Gouvernennent Minsk, Difrict Mazyrz (bei hokicki?) in Lithaucn einzeln ant dem Sande liegend

chen Sicllen wic nurezelonafsig krylkallifrt, mit dichtern inliegenden Stürken, wie eine Ilafelunfu, mitunter auch wio cin Apled gruli. Es liefs tich wie andies weiches Eifea licheniedtls. Hr. Henry liclt eb für kein Ofenproilukt, Conderu wiu viclmehr gentigl, es für melenrifih zu halen. Iis lielis lich nichls endiges oder verglaftes clarin crkennen; auch Guclen lich durt krino Eilenwetke, und aiches als ein elwas eifenhaltiger Wadir, das in dea Tara nielie Die Mal-le ifr vou den A rbeitern zeifchlagen wordea; (wäre fie racleorif:h gcwefen, lo wiirdul fie es woll aichl gehunnt hab(H); uncl was IIr. Jienry nit nach Florac gebracht hat, wog 25 Kilogrammen, (5u P[und). Nacb deu Ssïcken zu urthrilun, die ich ian Kial: Nisturalienkjbinet ,2u Wien daron gelelicn habe, and duen, die ich durclı die Güte des Hau. Paul Patfeh belize, halse ich es für ein Produke irbend ciner irdifcluen Schmelzugg, das lauge in Water gelegea hat, Die inliegenulen diehen Theile fiut melallifelies Eifen. Ao uneinen Stucke diefar Art, etwa ron der. Grölse einer Hahelnuls, Gud eckige ITervorragungen, falt wie an dem Pallexifclen Eifen, hier uad da unit KryfrallifacionsElächea, das übrige, welches Schwanmig ift, finile ich lehr deun Eilen ühnlich, das an manchen Ortea in Thuringen in kleinen Parthien gefelumolzen und erfi laernach uuter dan Hanzuer dichs wirit. Auch fehe ich in mauchen Höhlungen; ebus fo it diefea, etw.ı ron grünlicbgelbea verfehlacken erdigen Thoilea. Die zulsere ziendich diche Riu- 


\section{[ 50 ]}

gefunden luat. $\mathrm{Da}_{\mathrm{a}}$ an der Stelle Reifende häufig vorïber kommen, fo mülste, meint Hr. Norodecki, dicfe Eifenmafe lungft bekannt leyn, hätte fie fich inmer dort befunden; daher er geneigt ley, fie fïr neuern meteoril'chen Url'prungs zu halten. Hr. La agier zerlegte diefes litthanilche Gediegen-Eilen auf Erficlien des Hrn. Bronguiart, und fand zwar niclit alle Beftandtheile darin, die das Pallasilche Eilin charakterifiren, insbefondere gar kein Chromium und gar keinen Schwefel ; fand aber doch Nickel und Kobalt nur in einer etwas geringern Mange als jenes Eilen diele beiden Metalle entlialt. Es beftelıt nïmlich nach ihm dieles Gediegen - Eifen ans Lithanen in 200 Theilen aus 97 bis $9^{8}$ Theilen Eilen, und 2 'Theilen Niokel, dem etwas Kobalt beigernengt ift.

Gillert.]

5. Beiträge zu den Nachrichten von andern herabgefallenen Sublianzen.

(Fortletzung des Aufratzes Ann. B. 55 S. 249)

Nicht blos von faubarligen Materien in trockner oder feucliter Geftalt, londern fogar von Haa-

de ilt fchlackig und blafig, etrras der an der Aachener and an der Mailäadifchen Eileuraatre befincllich gewefeneu Rinde älnlich, nar unit gröfuern Blafen und mil weniger erdigen Theilen. Mau fact mir, diefes Lifon fey in Patis zitunlich thener vorkmuf wurden. Der beigefuglen Eliquelte zufulge liat Vaqquelin darin heiuen Nickel gefunilen und hale es fir niche meteorilch, fo wic es 2 el warten war. $c h l$. innsl. d. Phyfik, P., fij., St. a. J. 28 ig. St. j. 


\section{[ 5i ]}

ren, Glauer Seide und fchwarsem Papier, welche in Mafle niedergefillen feyn l'ollen, wird man hier Nachrichten finden. Wahrlelieinlich fund damit faferige und membranöle Stoffe gemeint, welche man in etwas diclen Dingen ahnlich gefunden liat, und es gehört viellcicht unter diele Kategorie auch dep von $L$ ivius erzithlte Niederfall einer dum Fleifche alunlichen Subftanz, welche zum Theil fchon in der Luft von Vögeln weggelchumppt worden, aber nicht, wie Fleilch, in Fulnifs übergegangen feyn foll. Was das für Stoffe gewefen find, und wo lie niïgen hergekonmen feyn, davon liefsen fich leiclit in Scherz munclierlei Erklarungen geben; aber es in Ernlte thun zu wollen, möchte wohl noch zu vorcilig feyn. Indeften halte ich doch für notlıwendig, diele Nachrichten zu erwähnen, denn wenn den Erzillulugen, die eben nicht das Geprige von Lügen - Berichten haben, etwas Wahres zum Grunde liegt, fo if doch wolll zu erwarten, dafs irgend ein Mal, frülier oder ljüter, fich etwas alunliches ereignen möclite, wo alsdann bei fchon vorlaunfig erregter Aufmerklinkeit eine beflere Unterliuchung der Sache zu erwarten if.

? 1592 den 5. Juli foll, nach Michael Baplt in C. Arsnei-, Kunf- und $U$ underbuch, 1. Tlicil S. go zu Rochlarufen, eine Meile von Eifurt, in eineu l'clirecklichen Gewitter, das einem Erdbcben gleich gewelion, vielen Scliaden angerichtet, und cin londerhares Getöle gegeben lıat, ein grolser Han- 


\section{$\left[\begin{array}{lll}3 & 55\end{array}\right]$}

fen einer dem Menfchenhaaren ahnlichen Subftanz niedergefallen feyn ").

Die einem Erdbeben ahnliche Erfchï terung, und das fonderbare Getôfe laflen nicht fowohl auf cin Gewitter, als auf ein anderes Meteor fchlielsen. Was es aber mit der herabgefallen feyn follenden haardhnlichen Subftanz für eine Bewandtnil's habe, ift fehr rutlufelhaft.

1623 den 12. Anguft 2wifchen 4 und $5 \mathrm{Uhr}$, (vermuthlich Nachmittags) *) war ein fogenannior Blutregen zu Strasburg, nachdem man vorher eino finftere, dicke, rothe oder rauchfarbene Wolke gefehen hatte, nach einem zu Strasburg 1623 gedruckten Auffatze von Il'a ac $\mathrm{Habrecht;}$ Bericht von einer fonderbaren Feuerkugel, S. 1, welchen ich in der Königl. Bibliothek zu Stuttgard fand.

1643 im Januar hat es zu Vaihingen an der Enz und zu Weinsberg fogenanntes Blut geregnet, nach einer handfchriftlichen Heilbronner Chronik aus welcher $\mathrm{Hr}$. Ob. Reg. Rath S ch übler in Stuttgard mir die Nachriclıt gefalligft mitgetheilt hat.

1652 im Mai hat, nach den Miscell. Ac. Nat. Cur. Dec. II. ann. 9. 26 o p. 120, Chriftian Mentzel des Nachts anf einer Reile swifchen Siena und Rom, als er aus dem Wagen geftiegen war, und zu

1) En iff darnuf das Chronodlfichon gemacht worden, BoChVsil Crlaes, Varil el typhone CaplLLI

IMPLVVIIs LapsI Larglter aelure; yola.

") Im Texte fielnt ausdrïcklioh Abends. Mollw 


\section{[ 30 ]}

Fufse ging, eine follr hello Sternfclunppe ganz in der Nähe niederfallen lielıen, die ilıren. Glanz bis ans Ende bohicht. Er fand an der Stclle eine durchfcheinende fcluleinige und klebrige. Subftenz, von der er eine Portion mitnahm. Sic ift hernach vertrocknet und hart geworden, und fein Solin, Cliurfürfl. Leibarzt in Borlin, hat fie hernach aufowalırt.

? 16 inj an grunen Domnerfiage, allo weil benerkt wird, dafs er in den Jalıre lélır zeitig eingatreten ift, in der sten Hailfte des Miriz "), foll boi Lancha nuweit Naumburg, 5 Meilen von Leipzig, eine Subfinz, wie dunkclblaue Ceidene Fuden, dcrcn Farbe mit den Aconitum Napellns verglichen wird, in grolser Munge nit dem Kegen oder Than herabgefallen feyn, nach Joll. Praetorius in cinemı zu Halle 1665 gedruckten Auflatze: Unerhör. tes Procligium von der heralgefallenen blauen Seide, welche ich in der Königl. Bibliothek zu Stuttyard angetroffin liabe. Die Faden follem ziemlich lang, und zmm Theil wis gedrelit gewelen feyn. Fis wurclen Thaler und Dukaten geboten, um etwas davon zu haben. Einige follen Hutbalnder und Schleifen davon getragen haben. Finc von Adel hatte viel davon gelanmelt, und es wollen $\Gamma_{\mathrm{p}}$ innen und in einer Kirche aufizingen lafen. Ganze Aecker follen feyn damit bedickt gewefen, und es foll an den Fülsen der Menfclien und Thiere hängen gebliehen leyn. Pratorius fagt, cr labe fulbli einiges

•) Dca 3.3. Arärz gămlich. Drollw. 


\section{[ [ $\left.\begin{array}{ll}37 & ]\end{array}\right]$}

davon gefchen, und der Pfarrer zu Cröbnitz, cine Jialbe Meile von Freiburg, labe einiges davon nach Halle gelchickt, einiges auch an Mehrere nach Lueipzig; es follen auch Einige von diefer Scide fich liaben Strünpfe ftricken laffen. (Was loll das wolıl gewefen fuyn?)

? $166 \mathrm{j}$ den 19 . Mai foll in Norwegen mit einem ungewöhnlichen Gewitter ein lechwefelartiger Staub gefallen leyn, der am Feuer liohr iibel gerochen, und mit Terpenthinöhl eine dem Schwefclbalfan ahnliclie Subftanz gegeben liabe, nucl Pauli de ufu Tabaci et Theae. (Kann vielleicht Blïthenftaub gewelen leynu.).

? 1686 don 31. Januar ift, nach Pli il. Jac.

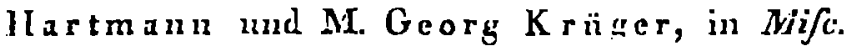
Ac. Nat. Cur. Dec. 2. ann. 2. pivo anno 68.9 in append. in Kurland in Ainbatfchen bei den Guts Rauden, dem Oberften Seefold gelıürig̨, einn L'chiwa!z.: papieraltige Subftunz in grofser Menge niederactallen. Sie foll mit Schnec und Sturm flockenweile hernbgekommen leyn. Fin grolser Platz an rinem Teiche foll des Moryens ganz lchwwarz überzogen gewefen leyn, und grolse' Stücke von der Grölise eines Tilches follen fingerlioch über einander gelegen haben. Hernacla loll es lievin vom Nordwinde zerriffen und in kleinen Stïcken unlher gefirent worden. Die Subftunz war foliwarz, als wenn fic wïle durclis Feuer geganigen. Der Geruch war anfangs faft wie Sec:tilit (d. i. von der See ansg'svorfene Arten von l'ucui. u. l' w., die dort zun Diunarn 


\section{[ 38! ]}

gebrapucht werdon,) An nianchon Stollen waren dio Blatter dinner, auf andern dicker, von der Confiftenz wio Lölchlpapies, aber folbwarz. Es hatton fich von der Stolle, wa fie gelegen hatten, Grashalino angehangngt, Mit Speicliel benetzt, furbte die Subftanz dic Finger nicht; fo liefs fich nicht zu Staub reiben, londern zeigte fich wio klebrige Hatutchen. Sie brannte hell, und glimnte wio Zunder, rock dabei anfangs falt wio verbrannt Papier, herzuach aber melir l'chwefelartig, aber lonft eben nicht widrig. Sio lieli etwas Alcho zurïick. Verdinnto Sulpeterfuure (Spiritud nilri) fral's die Subftanz niclit an, fondern orweichte fie nur etwal, und verüuderto dio lichware Farbe in roth. Alkalien vermelirton dio Schwdrze, bewirkten fonft aber keine Verunderung.

${ }_{1721}$ in der Mitto des Mürz muls ein rother Schlammregen mit oinem vorhergegangenon merkwürdigen Meteor fich um Sturtgrrd oreignot haben. Aun einem Soluroiben des damaligen Rentkonunifar und Expeditionaratlis Vifohor an den Steuterfokretair $S_{\text {flh }}$ it blar in Hoilbrunn, vom 91, Marz 373 hat nir Hr, Ob. Kog. Rath Scli üblor ins Stuttgard, der es unter leinen Familienpapieren befitzt, folgonden Auskug gefalligft mitgatheilt: "Allhier in Stuttyard if man, foit einer Waclie hor in giomlichar Boftüranug, sund zwar, dal' cin Pluznonien fich hier und da gezerigt lat, fo wio aus beigehenden Zettaluin all orfalien in," (Diefo Beilago des Briofeg falli jistat, to mag wahrfcheinlialı eine Zeiclunumg gowelion (eyn.) "Am folganden Tag hat os blut go- 


\section{[ is) ]}

regnel, fo dafs folches mit Hïnden anfgefangen werden können, und wo es lingefallen ift, kann es noch zum Theil gefehen werden. Gott la Te uns dadurch nichts Böfes andenten, und ftche uns bei."“

Zu der Nachiriclit von dem 1755 den 20. Oktober zwifchen 5 und 4 Uhr Nachmittags auf der Inlel Jetland gefallenen fcliwarzen Staube, der nicht voum Hekla gekommen if ; (Ann. B. 55 S. 259), füge icl linzu, dal's nach den Phil. Tiransact. Vol. 49 p. 5 ro bei filler Wittornng zwifchen Schetland und Island fchwarzer Stanb in folcher Monge auf ein Schinf zefallen ift, dals das Verdeck und das Tanwerk diclit damit überdeckt' worden find.

Von der 1796 an 8. Mïrz um $10 \frac{7}{6}$ Ulir Abends in der Oberlaufite nit einer leuerkugel lerabgefalIcnen bituminölen Subftanz (Ann. B. 55 S. 2:-2) laat Herr Dr. Baner, Arzt in Kleinwelka, auf die Verwendung des verftorbenen Bergratly Seyfert in Dresden mir etwas zukompen zu lallün dic Güte geliabt. Sie liat dic Confiftenz eines bramien Houigs, und es befinden fich noch $G_{\text {ras nud }}$ Heidekrantsblattchen darin, an denen lie angefeften lıat. Der Gernoh ift wie ein etwas verdorbner Oelnlfirnifs. Hutte ich molir davon, l'o würde iclı es gern zu einer clionifchen Analyle beftimmen, indeTen glaube ich nicht, dafs man andere Beftandtheile darin finden würde, als Schwefel und Kohlenfioff, vielleicht mit einer geringen Beimengung von Kielelerde und Liilenoxyd. Das belte Auflülungsmittel möchte meincs Liraclitens dor logenannte Schwofel- 


\section{[ \$0]}

Alkolıol von Lanpadius leyn, mit welchen dicle Subftanz einige Verwandifchaft zu haben l'clucint.

${ }^{809}$ im April, rotler Regen in der Ghiara d'Adda im Ventianifihen, "nach $L$ u ig i B ol'l'i, in Giorn, di fif. e chin. 1818, ado buneftre ग. $209 . ")$

1814 in der Nacht vor den 28. Oktober fiel in Thale von Uneglia nicht weit von Genova ein Rcgen von rother Erde. Sis war weicl und fein, behiclt das Wafter lango in fich und fchien thonartig zu fnyn. Es waren auch weilso und feliwarze Körnchen darunter; erftere waren l'climmernd, und braulten mit Salpotediure. Lavagna, der davou cbendal'elbft p. 52 Nachricht gicbt, bunerkt, dal's es nicht von Inlecten herrülıren könue, wic Valnont do Bomare angenommen hat; er ift aber genoigt, es durch cinen Wirbelwind aus Afrika lıerüberfülıren zil lafen. Er bemerkt, dalo vor 60 Jaliren etwas ulinliches Statt gofunden habe.

18ı6, den 15. April fiel auf dem Berge Tonale und an einigen andern Orten im nördlichen lialien all rothen Wolken ziegelrother Sclinec, nach denl'elben Giornale 1818, p. 475. Dox Bodenlatz gab ein erdiges Pulver, lolir loicht und fein, etwas fettig auzufüllen, von dunkelgrauer l'arbe, das thonig roch, etwas falzig und zuliommenzichend l'alıneckte,

") Der nach den Ann. de Chim. 8. 9 p. 216,2812 im Mai nuf Barbaclos niedergefalleue grünliche Sinub full vom Vulkan

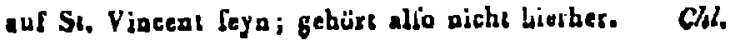




\section{3}

und nicht rom Muguut angezogen wurde. In 26 Gran fanden fich bei dor Analyle, Kiclelerdo 8, Eifun 5, Alanuerde 5, Kalkerde 1, Kohlenläure z Sclivefel $\frac{x}{4}$, brenzliches Uchl 2 , Kollenftoff 2, Waffor 2 Gran. Der Verlufi war $2 \frac{1}{4}$ Gran. Schade, dafs run es nicht auch auf Chrom und anf Magnefia unterluclut hat. Es wird für etwas von den Motcorftcinon galuz vorfaliedenes orklärt, (wohl nicht nit H(cht), anch behauptet, lulche Erl'chemungen kimen nur in der Früllingszcit vor, (welches umgegrïnklet ift, da ich lier und anch in Alunal. B. 55 S. I'g etc. genng Beilpiele ans andern Jahriesciten ge'Leben habe etc.), und dem zu Folge vermutlic, dal's cin Aequinoctiallium dicli-s rotl:a Pulver. aus Afrika herbeigefilirt liabe.

${ }_{1} \mathrm{U}_{1} 8$ fand Kapitain $\mathrm{R}$ of $\mathrm{s}$ rothen Schnee an der nürdlichen Küfte rler Baffusbay, md bruchte clwas von den Bodenlitze mit nach Enjolude. Vielleicht erbalten wir bald cine Analyle davon *).

-) Eine klelne Menge dinfer rodlen Schnees, welche; durch Kapilain Saabye und Hrn. Biol an Hrn. Then ard gekomenen war, ill ron dielum Chemiker in der Abliche unterfucht wurlien, nin anbanmachen, ob der farbende Kürper wirklich thicrifeleen Lifprunge fuy, wie man anfangu glauhie. bei der acrfiiitentlen Delillation enefend keine Spur rou kohlenfaurein smmoniak, wie et hätte feyn mülri-a, wäre liefes der Full gewefen, dagegrn breazliclies Oeht und eine foste Minterie; der rolilfisbende Kärper feheiul alfo deın Dilanzenreiclie angehïrt $2 u$ habea, wio licb aucb dadurch bnfiüligte, dals eine Aultöfung dilfrbeo in Alkohol nack 


\title{
[ $4=3$
}

Vou rothem Schuce hat auch in Sommer ${ }_{1} 8_{18}$ Hr. Jolann von Cliarpentier, Salinendirektor in Bex, den iclı als Freund eben fo lehr, wie in wilfenfchaftlicher Hinficht achte, auf der Alpo Anceindaz, welche an Fufse der Dinblerets liugt, und an andern benachbarten Stellen einiges gefunden, und mir ctwas voiı dem nach dem Aufthanen übrig̨ gebliebenen Rückftande zu iberl'chicken die Gütc gehabt. Er ift dunkel - rotlibraun, oder graulichbiaun, fein anzufülulen, und euthïlt, eben fo wic vou dem 1815 und 1814 gefallenen gemeldet

\begin{abstract}
Abdampfeu des Alkobols einen retligen Kurper zuruekliefs; was Gich in Alkuhul niche auggelifí liatle fchien erdig a fejn. - In piner Fldiche voll gefumuelzen rohen Schnecs aus der Baflinslay, welcleo Hr. Decan do le aus Englad mit uach Paris brachie, hatte der rallifirbencle Kürper bjuncu a Dloualen keinu Verinderung gelituen; er beftulu aus rothen Kügelchen, die nach Dr. Wollaftun's MeIrung g zós bis rơै Gull Durchinefrer laben; von den kleiulken Gnd manebo weifo und aueinander geklebe und hahen dan ein bilutiges Ausfehen. Geprefut follen lie brinen pulverulenten, fondera einen rö̈hlichen Staligen Kïrper geben, und eise Earbenlole IIane a arücklaITen, leheinen abor heise Champigaops, Condern, ric dehon Ilr. Bunu in Antange zu Kapitnı llof Reifeberichıte vermullete, kleine, zur Faruilie iler Algen gehïronde l'llanzenkürper, dem Saamen der Ulsas alınlich zu foyn. - Eis, das ficb iic vorigen Winter in einesn Gruben eines in der Gugend von Lupzig liegenulen Laniguts gebildet hatte, zeigte an Teidei Oberï̈che eine rolearotb gefïrble Lage, deren vegetabilifelaor Urfprung kaum aweiftiluph zu leyu rebien. Gilb.
\end{abstract}




\section{$\left[\begin{array}{lll}\mathbf{4} & \mathbf{5}\end{array}\right]$}

wird, einzelno gröbore den Pyropen shnliche Körncluen. Etwas davon habo ich dem Hrn. Hofrath Strouneyor in Güttingen zur Analyle überlchickt. So bald ich weitoro Nachricht davon erlaalte, bin boreit, mchreres davon bekannt au maolicn.

4. Nene Nachricbien ran Fonermeleoyen, deron Milton mas diclst hablaft gewordea iti "J.

2813 den 37. Januar, um 8 Ulır 12 Minuten (Vormittags oder Abends?) zeigte fich ein unbederttendes Wölkchen in SSW, etwa 12 bis $13^{\circ}$ hoch, welches fich lichnell kroisförmig bewogte und angenblicklich in einen Fenerball ausbrach, deffen Durclıznefler auf 16 gel'cliňtzt wird. Dieler Ausbruch war von oinem Gerünlcils hegleitet, als worn Waller anf glühendes Eifen gcgollen wird. Der Feuerball ber*

1) Von awei altoru giebs O learil Holygraphia Lelpzig uGriy solgeade, rau \&lira. Prof, M eineck a mir milgatleilte Nacli-

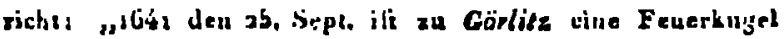
vau Hiramel unter dio oluurfurfilichen und kaiferl. Saldatea gefallor," heifst es S. *1fi - und sa 1665 den 9. Juli has man in Laipzig vun einer Feuorkugel, fo vam Hirumel gafallen, gefagia" S. 486, (deinfulbea Werke, Welchea voa dem bekanntou Friodehurger Steinpryen S, ${ }^{5} 7$ meldst: "Im Julir 1304 find in einesa Munaerwenter hei Friclebarg on der Saalo glühoull hrifyo Steiuw horab gefallen, wolche kolul-

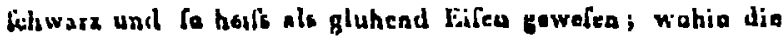
gufallen, hibea fo das Girs gleich, als wean Kablfeuer dasiiber gowrius, versengt."; Cill, 


\section{[4;]}

fiete, und war von cinem pritutig leuchtenden Scliwcife begleitet. Er ficl (ocler ging weiter) jur einer Diagonale von WSW gegen SW: Das Horizont war felur crleuclutet. Dic Erfclucinung daucrte 5-Sekunden "). Hicr ift allo die erfte Ankunft und Enizindung einer lolclicu Malle bcobschtet worden, welclic fich auch nool bei cinigen andern Meteoren l'o yezcigt hat, bei manclien aber auch wic eine l'cluncll ficlutbar werdende ficle nach und nach vergrël'scrucle Stcrnl'clinnjpe, oiler auch, win cin oder nelurere parallele diblultiecifen, aus welchi'n fich fchnell cin ftirker lonchsender und brennenter liörper vildete, welelies nuftucilig davon abliningt, ob eine Jolchic Minle melur locker ausgedelunt, oder zufannengebsllt, odes lelur in die Linige gezogen in unferer Atnolphinic sukonme.

281:' den 28. Oktober un 7 Lihr jo' Abends,

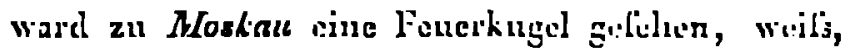
grol's wie der aufgelende Vollmond, fie ging von $N$ rach S, langlaner, als gewölunlich die Sternlelunuppen, Sie l'chien fich un dic Axc 21 drelien. Einige liaben fie behaart Eelelicn, (wolul ganz richtig, wegen der ausbreclicnden Flimnen und Dïnrf(c) *).

-) Aus eillem Beriehie von II allalehk a, Prof. Jer Phyfik in Brunn, in Helperus $2 \gamma_{1}$, No. 2t. S. 122.

") Niehriclıten durov giehe Fi feher, Direksor der nalurfurCchondea Gefullehofi zu Muskau, in den Mem. de l't: Inij. da st, Kaferslon'g, t. T, hili. p. Sn. 


$$
\text { [ } 5]
$$

Aus dem Jalıre 2816 werden in den Naval Chronicle a grofse Metcore, etwa halb fo grols als der Mond, und 18 kleinere angegeben. (Journ. of fc. and arts, Scpt. p. 132.)

In verwichenen Jalire 1818 find zienlich viele Fenermeteore beolnachtet worden, von denen mir folgende bekannt worden fincl:

${ }_{18} 8$ den 18 . Januar gegen 8 Uhr Abends, iff, nach Zeitungaberichten ans Petersburg, zir Turruchanst in Sibirien (bei $57^{\circ} \mathrm{Kalte}$ ) ein inmer anwachlendes kirachen in der Luft gehört worden, licrauf (foll wolhl heifsen: zugleich, oder vorher) ift cin lchlangenförmiges (allo ancl wie lo viele andere, in Bogenfprïrngen gehondes) lenemeteor erfchienen und bald verichwwunden.

1818 den 28. Januar um 6 Ulir Abonds, ift ein Feuermeteor mit cinem langen Schweife gefeluen worden zu Camplell-Town bei Fort St. George $*$.

18,8 den 6. Februar zwilchen 2 und 3 Uhr Nachmittags fah man in England an mehrem Orten ein, ungeachtet des Sonnenlichts, fehr helles Feuerneteor, da:s fich fehnell vom Zenith gegen den Horizont nach $\mathrm{N}$ bewegte. Es fchieu in ciniger Höhe,

-) Journal of fcience and art, No. 9. p. 233 . Wenn gefigt wirrl, der Durchmeftur lay I Fufs grafs, und des Schweif 6 Fulf ling erfehienen, $\delta_{0}$ ifi dirriu kein Siull, weil man nicht wifren kann, in welcher Eatferung, oder unter welchem Winkel der Firzäbler fich dirfe Grüfsen deuke.

Chiluss:. 


\section{[ 46$]$}

elwa $15^{\circ}$ über dein Horizont, zu verfclw winden, und man lah liernach cine Dunkellıcit (cine zurückgelaflene Raucliwolke) nach $N$ etwa $12^{\circ}$ hoch. An melirern Orten, z. B. zu Swaffliam in Norfolk, zu Coningby in Lincolnfhire, zu Holdernel's, Tren!fall (5o englifclue Mcilen von Coningby) u. [: w: hürte nan ein Getöle, wio von vielen Wagengorallel, und verfpürte eine Erl'cluñlterung einige Sekunden lang, wie bei einem Erdbeben. Thoml'o n's Ann. April 1818 p. 273 . Journ. of fc. No. 9 . p. 132 und 155 .

Dal's das 2818 den 15 . Febr. um 6 Uhr im lïd. weftlichen Frankreich gelehene Fenerneteor, wovon in Ann. B. 6o S. 252 melıreres gelagt worden ift, bei Limoges eine grolse Vertiefung in die Erde gefeluagen habe, foll, vic mir gemeldet worden, ungegründet leyn.

1818 den 27. Juli Abends zwifchen 9 und 10 Ulir v'urde in Amerila in dem Dorfe Vermont bei Montpellier ein glănzendes Feuermeteor gedchen, dns er fi nach $O$ l'chuell fenlirecht niederwarts, und lodann (licheinbar) horizontal nordwärts (allo auch wie fo viele andere, in 'Lickzackl'prüngen) ging. Es erfchien grols wie der Vollnıond, birnförmiz, das breitere Ende nach der Erde gekelirt. Es hatte das Anlehen eines foliclen Kürpers. Unmittelbar darauf folgten a kleinere Feuerkugeln. Man verglich das Licht mit glühenden Eilen. Nach 2 oder 5 Minuten, nach Andern, mach 4 bis 5 Minuten, hörte man heftiges donnerartiges Getöle, welclues Einige 


\section{[ 47$]$}

unit dem Rollen eines Wagens anf Steinen vergliclien, Journ. of $\int c$. No. 11 p. 1 tio.

18,8 den 5. Ang. un 11 Uhr Abends fah $T$ hona a Y o un g ( Sekretair der Kön. Societut zu London) zu Whorthing (in $50^{\circ} 49^{\prime}$ Breite nnd $20^{\circ}$ weftlich von Grenwich) ein lehr helles Meteor bei der Cafiopea, welches nichto anders als der nachgelatene Schweif einer Fenerkugel kann gewefen feyn. Es war cin Lichtireif, der in $19^{\circ}$ Polardifianz und $65^{\circ}$ Rectalcenfion anfing und in $17^{\circ}$ Polardiftanz und $80^{\circ}$ Rectafcenlion endigte. Er blicb eine Minute lang ficlubar, olune Bewegung, faft wie cin Komet. Ann. de Ch. t. 9 p. 88.

1818 den 5. Aug. um 9 Uhir 10', lah man über die Stadt Chelmford in England cin lchı̈̈nes und grofscs Fenermeteor nach NO anfserordentlich fchnell gelien. Journ. of $/ c$. No. 11. p. 160 .

1818 in der Naclat vom 5. bis 6. Sejt. fal man nach den Zcitungen zwilchen Mitternacht und \& Uhr 212 Breteuil in Uiledepartement eine Fenerkugel von $W$ gegen $N$ in eincr krummen Richtung guhen; fie zerplnizte gegen $\mathrm{N}$ in mehrere leuchtende Stücke mit Gutölis.

1818 den 14. Sept. Abends un $20 \frac{1}{3}$ the wurde in England ein Fenermeteor in einer mäfigen Höhe über dem Horizont erblickt, welches nach $N$ ging:Es crfclien lo grofs wie der.Mond mit einem erft rothen, dann weilsen Schweife. Thom 1 Ann. Olkt. 1818 p. 530 .

818 den 3ı. Olitober, um halb ncun Lilhr 


\section{1 iji $\rfloor$}

Abends, falt man bei fouft heilcrn Himmel zu Mlehadia in Banuat in SO cinc fenriģ unfờnuliclıe Malte, welche heruach cine linngliche Form aunahnu, und in luanfige zur Erde fallende Funken, ohne hörbareu Knall (vermulhlich weil der Ort der Explofion zu entferut wrar), lich auflöfte, und die gazze Gegend in ein leclles 5 Minuten lang dauerndes Feucu zu verfutzen fchien. Man fah hiurauf eine belenclitete beinalhe feurige mit cinen fchwarzen Flek-

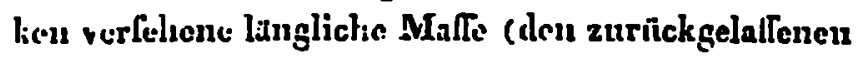
2um Theil noch leuclitenden Nanip (des Meteors), welche, nachidem fie in der Mille gleichliun alogebrochten, zwci unförunliche noch immer Jeuchtentes Haiften bildete, die allmulhlig imner kleiner wurden und endlich verfeliwanden ").

1318 in der Naclit rom 22. zum 32. Decembrrr faln man in Fulunen gegen SSW ein Meloor vic cinen Step'n, von der Grölse des Mondes mit einem Dumfikreile, aus welehem wahrend melurerer Sckunden (allem Anlehen nach durch cisen Druckfeliler

-) Wherer Zeitung ucm 10. December afis8. Niteh audein Machichten trabea auch Couriere und andere Reifende zwiTchen Jally uad bukarelt uogefahr datholbe gatehen. Miclleiche bukounnen wir in der Folge ron niederyefnllenen Maflen, etwe in Bulgatieu viler Aumelica, eines türkifehen Borjeht, wie war aleren fehon einige holver, die uirht unler lue felutchtelien gel.ijıen, weil cin Tulke wenigliens das teulich wieller erzilalt, was er grlichen hat, alser munchics Phy ther gern Ieine vorgrfaliten Mtinusugen in die Erashlugg rou Thathehes hineintrugt.

chl. 


\section{[ 496$]$}

heilst es: Stunden), \{kleine Sterne hervorgingen, den fogenannten bölımifchen Lichtern nichıt unallenlicle *).

\section{Z U S A 'T Z $\mathbf{Z}$.}

Noch unbenutzte chinefif:he Nachrichten von Meteorfteinen.

Frei ansgezogen aus einem Aufratze des IIerrn

J. P. Aber-Remusat "*).

In den Jahrbüchern der Chinefen und der Japaner finden fich manche Nachrichten vom Herabfallen von Meteorfteisen. Es foheint mir, fagt Hir. Remulat, der Mühe werth zu feyn, die Umftände zufaminen zu ftellen, unter welchen diefrs dort gefchah. Die gewühnliche Benennung der Mreteorfteinc ift: fing yun tich/hing chi, das in herabfallende, in Stein verwandelte Sterne. So heifsen fie blos, bemerlet ein chinefifcher Schrifteller, weil fie firh dex Augen wie Sterne zeigen; Steine aber wirklich für Sterne zu halien, würde, fagt er, ein grofser Irrthım feyn. Denn, bemerkt

-) Nach ciner Zeilongsnachriche, unter aodern im Korrefpiondenten yon und für Deutfikland 1319 , 10. St. vom luten Jauluar.

-) Im Journal des Savans und in len Ann. de Ch. et do Phyf. des Hra. Gay-Iuffac und Arago t. 10. 23 ig. Gilb.

Aunal, Il, Pliglik, B, 63, St. 1. J. 18 g St.g. 


\section{[ 50 ]}

ein anderer chivefifcher Schriliftelier, es find feit Altors unzählig vicle folcher fclueiubareu Sterne herabgefollen, und die Zahl der Sierne hat lich doch nicht vermindert; auch find fie nach ibm felten über cinen $t c / b / i$ und einen $t / h / \iota n,(0,+19$ Meter) lang. Dafs es indefs viel gröfsere gegebeu liabe, dafiir fülurt Hr. Remufat das Beifpiel des an der Quelle des gelben FlufJes, an der Nordfeite des Altan. fich findenden Fulfen an, welchen die Mongulen Khada foutfilav, (Fulfen des Pols) nennen, und von dem unter ihnen die Sage gcht, er fey ein herahgefallener Stern. E. ift über tchang (ungefilhr is Meter) hoch, Steht mitten in eiuer Eluene ganz einzeln da, und Hr. Remufat vermuthet in ilua cine IIaffe gediegenen Eifcos, vou der Art derer von Krasnajarsk, von Otumpa, von Mexiko u. a. Uebrigens haben die Chincfen noch Hrn. Remufat auch dic Sugen von haches de foudre, lifjoirs, marteaux, coins, vitlles, annequx, perles de foudre, oder viclnebr du dieu du tonnere, von denen ein chinefifcher Schriftfteller behauptet, fie fejen von derfelben Natur als die herabfalleaden Sterne, und Veberrefte von ähnlichen Erfcheinungen, als Stein-, Gold-, Hirfen-, Reifs-, Haar-, Blut-Regen und derbleichen muhr, die in den Chroniken vorkommen.

Mehrentheils find die chinefifchen Neteorfteine nis Fetterkugeln angekommen. Maochmal hat man aber ditfe nicht benuerkt, und if plötzlich von den mehrere $100 \mathrm{Li}$ (10 eine Lieue) wcit zu hörenden, donnerähnlichen Getöfe iibcrafcht worden, wäbrend deffen die Steine manchmal bei gauz heiteru Himmul hesubficlen. Das Getöfe beim Zerplatzen vler Fenerkugel vird mit dem eines einftürzenden Haufes. 


\section{$\left[\begin{array}{ll}5 x & ]\end{array}\right.$}

dem Gebrüll eines Stiers, und das Gctöfe des Herabfallens init dem der Flügel wilder $G a ̈ n f e$ verglichen. Es find einer oder 2 wci oder mehrere Steine herabyekommen, manchinal hat es wie Steine geregnet. Die Stcine waren beim Herabfallen brennend heifs, fchwärzlich, natuchmal fehr leicht. Ein Schein von ein ger Ausdehnumg, der mit einer Schlange verglichen zu werden und einige Zeit zu beftehen pflegt, zeizt fich an dem Orte, wo die Feuerkugel war; der Himmel ift dort bläffer, manchmal gelblich roth oder grünlich, wie Dickicht von Bambusrohr. Es find Steine herabgefallen in Ackerfeldern, Lïgern, Städten, und in der Hauptftadt, und mehrmals find Thicre dadurch in Schrocken gefetzt worden. Als im Jahr 546 ein folcher Stein in dem Lager von Kao - tfou herabfiel, fingen darin alle Efel an $z u$ fchreien. Unter Chi-tfoung, einem der fpätern Tcheou, fiel ein Stcin unter grofsem Geräufch nahe bei der Hauptfladt herab; Pferde und Nindvich entflohen, ohne dafs inan fie halten konnte; in der Stadt glaubte man es tronunele und man fing auch in Scblofe an zu trommeln. Es kummt kein Beifpiel var, dafs ein Menfeh ron Meteorfteinen getroffen worden fey.

Ich liabe, fagt Hr. Remufat, ein Verzeichnifs der in China herabgefallenen Meteorfteine, und der Umftände, unter denen diefes gefchah, nach Ma-touan-lin gemacht. In einer Art chronologifcher Tafel, welche Hr. Deguignes, der Sohn, in den erften Band feiner Reife naek Peking eingerūckt, wahrfeheinlich aber nicht felbft gemacht hat, wcrden 9 folche Erfcheinungen aus dor Zeit vor Chrifti Gebut erwähat; diefe Auszüge aus den ziemlich unftändlichens Errzäblungen der Chinefen find aber zu unvollftändig und zu 


\section{$\left[\begin{array}{ll}52 & ]\end{array}\right.$}

fummarifch. Das Verzeichnifs Ma-touan-lin's figtt zu den uns fchon bekarmten Fällen, über 60 netue hinzu, und geht doch nur bis zu dem Jahre 1004. Ich habe dicfe chronolugifche Folge aus andern Quellen fortzufetzen mich bemuilt. Hicr cinize Beifpicle der neueften Fälle, oder folcher, welche etwas Befonderes haben, damit man heurtheilen könne, ob das ganze Verzeichnifs bekannt gemacht z.u werden verdiene.

Im fechiften Jahrc Youan-ho $(811)$, im dritten Mond, am Tage $W n u$ - fiu, zwifchen 3 und 5 vhr Nachmittags, fah man hei bedecktem Himmel und kaltem Wetter, eine Fencrkuyel fo grofs wie ein hou (ein Gemärs 10 Boiffeaus haltend) welche zwifchen Yan und Yun herabficl. Man hörte mehrerc $100 \mathrm{Li}$ weit ein dem Donner ähnliches Getöfe, und die Fafanen flogen mit Gefchrei fort. Leber dem Orte, wo die Feuerkugel herabficl, blieb ein röthlicher Dampf, von der Länge eines Tchangs ( 3,8 Meter) wie eine Schlange aufigerichtet und dauerte bis an Abend, worauf ex verlöfchte.

Im 2 wöllten Jahre ( 817$)$, im neunten MIond, am Tage $K i-K a \ddot{i}$ um 3 oder 4 Uhr nach Mitternacht, erfchien um die Mlitte des Himmels ein tliefsender Stem (étoile conlante); der Kopf war wie ein Eimer, der Schwanz wie ein Kahn von 200 Hou Laft; der Stcrn war über 10 tchang ( 38 Meter) lang, machte ein Gerïufch wie ein Schwarn Vögel, der davon fliegt, und hatte ein Licht wic Fackeln. Der Stern zog nach Weften unter dem Mond fort; plötzlich hürte man ein grofses Cetöfe, und in dem Augenblick als die Kingrl zur Erde fiel, einen drei Mal fo Itarken Lärm, als wunn cin Hitus einflurzt. 


\section{[ 55 j $]$}

Im zweiten Jahre thian-yeou ( 905 ) in dritteu Mond, an Tage $I$ - $t c h e o u$, ñegen Mitternacht, erfelicn nitten am Firmament cin grofscr Stern, 5 Boiffeaux grols. Er floss ungtfithr 10 Tchang weit nach Nordweft, und hlieb dann ttehen. Es waren uber ifm eine Menge klciner Sterne, die $\mathbf{n}$ ic eine rothe oder orangelarbene, wenigftens 5 Tchang lanje Flamme, die fich wie cine Schlange hinzog, bildeten. Alle diefe kleinen Sterne bewegten fich nach Sudoft und fielen danu als ein Regen lieral,; Jald darauf verlufehte clie hugel und es blieb blos ein weifslich hlaucr ins Grunliche fpiclender I)unft, der dit Hitte des Hiramels eimnahm, ficls verdunkelte und verSehwand.

In den Jahien Wan - li der Dynaftic der Ming $(15,6)$ in zwolften MIond, am 2.5. "Yagc, liefs fich zu Chun-khing. fou in der Provinz Sse-lch/toucan, als weder Wind norb Wolken da waren, plutelich ein Donnein huren, und es fiel.1 6 runde Stcinc hrah, welche 8, 15, 17, die bleinern utu 1 Pfund, die kleiuften nur 10 Unzen wogen.

Unter der liegierung des Künigrs von Korra W en-tfoung, welches nit dem zwriten Jalure thyan-veou (905) ubercinItiunt, ficlon zu Hoang-lie in Korea Steine mit eincm dunneralinlichen Getufe liciab, welche die dafigen Officiere an clen Hof fohichten. Dur $\mathrm{P}_{1}$ ifident des Ceremonies benterkte in einer Supplik an den kunig, diefes Ifcrabfallen von Sterweil habe fich fchon unter den vorigen Dynattien mehronals ucignet, und fey daher rnichis Aufserurdentliches, und hein Whuder, das ant Gluck oder Unuluck deute, und man thue rimccht daruber au erftanen.

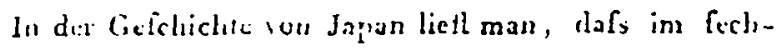




\section{[ $5: t]$}

Sten Jahre, fiowa, der Hcrrfchaft von Nin-Mio-Ten-O (839) am 29. Tage des 8. Monds weftlich von der Stadt T/lian-tch/zouan es angefangen habe $z u$ donnens und $x u$ regnen, zehn Tage lang, und dals, als es wieder helles Wetter wurde, man an diefetn fonft Steinlofen Ortc einige Steine Feilfpitzen und rothen Aexten khnlich gefunden habe.

Aeholiche Beifpiele follen in 2 wel andern Städten Japans doch 3 Jahro hintereinander vorgekonmen feyn, unter der Regierung von Kouolio-Ton- $U$ in den Jahren nenwa, daz heifst 885,886 und 887 . Der japanifche Schriftetellev behauptet, in den nürdlichen Idündern felen weil ¡hüufiger Donnerfteine herab, in Japan aber nur felten, wovon er als Beweis eins der fürchtcrlichiten Gewitter, die es je gegeben bat, unfuhit, welches an 20. Tage dea G. Monds im J. 1710 iiher die Ifauptftadt Japaps losbrach, Es fchlug an viclen Orten ein, der Blitz zest ftürte mehrere Hunderto von Häufern, und doch fand man anch nicht eine jener angeblichen Aexto pder keile des Donnergottes.

So weit die als Lisn. Remufal's Auffata ausgezogenen Nachrichten aum Clina und Japan.

Gilbort.] 